\title{
Recurrent cardiac events in patients with idiopathic ventricular fibrillation, excluding patients with the Brugada syndrome Jean Champagne*1, Peter Geelen ${ }^{1}$, François Philippon ${ }^{2}$ and Pedro Brugada ${ }^{1}$
} \author{
Chemin Ste-Foy, Quebec City, Quebec, Canada \\ Email: Jean Champagne* - jean.champagne@med.ulaval.ca; Peter Geelen - peter.geelen@wol.be; \\ François Philippon - francois.philippon@med.ulaval.ca; Pedro Brugada - Pedro.Brugada@scarlet.be \\ * Corresponding author
}

Address: ${ }^{1}$ Cardiovascular Center, OLV Ziekenhuis, Moorselbaan 164, 9300 Aalst, Belgium and ${ }^{2}$ Quebec Heart Institute, Laval Hospital, 2725,

Published: 0I January 2005

BMC Medicine 2005, 3:1 doi:10.1 |86/|74|-70|5-3-1
Received: 18 August 2004

Accepted: 0I January 2005

This article is available from: http://www.biomedcentral.com/|74|-70|5/3/I

(C) 2005 Champagne et al; licensee BioMed Central Ltd.

This is an Open Access article distributed under the terms of the Creative Commons Attribution License (http://creativecommons.org/licenses/by/2.0), which permits unrestricted use, distribution, and reproduction in any medium, provided the original work is properly cited.

\begin{abstract}
Background: The recurrence of cardiac events in patients with idiopathic ventricular fibrillation (VF) excluding patients with the Brugada syndrome is unclear since this entity remains present in previous studies.

Methods: Since 1992, 18 patients (72\% male) with idiopathic VF out of 455 ICD implants were treated with an implantable cardioverter defibrillator (ICD). The mean age at first ICD implantation was $42 \pm 14$ years. Brugada syndrome, as well as other primary electrical diseases (e.g. long QT), were systematically excluded in all patients by the absence of the typical electrocardiogram (ST elevation in the right precordial leads) at rest and/or after pharmacological tests (ajmaline, flecainide, or procainamide). Recurrence of cardiac events was prospectively assessed.

Results: During a mean follow-up period of $4 \mathrm{I} \pm 27$ months, VF recurrence with appropriate shock occurred in 7 patients (39\%) covering a total of 27 shocks. The median time to first appropriate shock was $12 \pm 9$ months. There were no deaths. In the electrophysiological study, $39 \%$ of patients were inducible, but inducibility failed to predict subsequent arrhythmic events. Forty-four percent of patients suffered 21 inappropriate shocks, which were caused by sinus tachycardia, atrial arrhythmias or lead malfunction.

Conclusion: Idiopathic ventricular fibrillation patients have a high recurrence rate of potentially fatal ventricular arrhythmias, excluding patients with the Brugada syndrome or other known causes. ICD prevents sudden cardiac death but inappropriate shocks remained a major issue in this young and active population.
\end{abstract}

\section{Background}

Idiopathic ventricular fibrillation (VF) is defined as spontaneous ventricular fibrillation in the absence of any structural heart disease, including coronary artery disease, valvular heart disease, myocarditis, cardiomyopathy or electrophysiological diseases, with a well-defined cause, such as the long QT syndrome, the Brugada syndrome, ventricular pre-excitation (WPW), and drug intoxication [1]. The consensus statement of the joint steering committees of the UCARE registry of Europe and of the idiopathic VF registry of US reported in 1997 that patients with the Brugada syndrome should be considered a variant of idiopathic VF [1]. 
Brugada syndrome is characterized by a unique electrocardiographic (ECG) pattern of right bundle branch block with ST elevation in the right precordial leads. Transient forms of the disease in which the ECG normalizes for a period of time have been described. Administration of sodium channel blockers will unmask the abnormal ECG in patients with transient normalized ECGs [2,3]. The American Heart Association has recently proposed diagnostic criteria for the Brugada syndrome [4]. Moreover, the incidence of Brugada syndrome among patients with idiopathic VF remains unclear. The prevalence of a Brugada type ECG pattern was reported in 21\% [5] and 24\% [6] of idiopathic VF populations. However, on the basis of pharmacological tests, it has been speculated that up to $40 \%-60 \%$ of patients diagnosed with idiopathic VF might actually suffer from the Brugada syndrome [7]. Since this is a new clinical entity, older publications probably classified some patients with Brugada syndrome as idiopathic VF, since intravenous administration of a sodium channel blocker to unmask a concealed form of the disease was not systematically performed. Our report describes the clinical and electrophysiological characteristics of consecutive sudden cardiac arrest survivors from idiopathic VF who received an ICD, and in whom electrical and structural heart diseases including Brugada syndrome, long QT, arrhythmogenic right ventricular dysplasia, and hypertrophic cardiomyopathy characterized by a high recurrence rate, were systematically excluded.

\section{Methods}

\section{Patient population}

The total population under investigation included 455 consecutive patients who received a third generation ICD, with stored electrograms capabilities for hemodynamically poorly tolerated ventricular tachyarrhythmias, from 1992 to 2000 . Of these, 29 were initially diagnosed as idiopathic VF associated with a structurally normal heart, normal left and right ventricular ejection fraction and normal coronary arteries. During the follow-up, 11 patients developed a new diagnosis potentially explaining VF, and were excluded from the final analysis. Four had right ventricular dysplasia, 5 had Brugada syndrome with the typical ECG manifestations after a drug challenge, and 2 patients had long QT syndrome. A final diagnosis of idiopathic VF was made in the remaining 18 patients. All patients had survived either an episode of cardiac arrest due to VF (12 patients) or a syncopal episode associated with documented self-terminating polymorphic ventricular tachycardia or fibrillation (6 patients). In 15 patients, the documented VF was seen during daily activity (one episode during sustained effort) and in 3 patients, during night-time. Out of the 18 patients, 10 had a previous history of unexplained syncope.

\section{Definitions and investigation}

Idiopathic VF was defined as VF in the absence of demonstrable cardiac abnormalities as previously reported [1]. Thus, we excluded other known causes of VT/VF such as WPW syndrome, congenital long QT syndrome, shortcoupled torsade de pointes, catecholamine-induced polymorphic VT, and Brugada syndrome. No patient had a past history of ischemic heart disease, congestive heart failure or family history of unexpected sudden cardiac death. All patients had a normal physical examination, blood testing and exercise test. Structural heart disease was excluded in all by echocardiography, cardiac catheterization including right and left ventricular angiography and coronary angiography. Patients on anti-arhythmic therapy before their cardiac arrest were excluded from the study, as well as those with electrolyte disturbances, history of significant alcohol or drug abuse or prolonged $\mathrm{QT}_{\mathrm{c}}$. Of the 18 patients, 10 patients had normal right ventricular endomyocardial biopsy and 8 patients had a normal cardiac magnetic resonance imaging. Moreover, in 5 patients, an ergonovine provocation test was performed to exclude coronary artery spasm. Brugada syndrome was systematically excluded in all patients by negative serial ECG recordings with the typical coved or saddle shapedtype ST-segment elevation in the right precordial leads. All patients received $i v$ administration of a sodium channel blocker to unmask a concealed form of the disease and all tests were negative. Flecainide ( $2 \mathrm{mg} / \mathrm{kg}$ body weight) was used in 9 patients, procainamide $(10 \mathrm{mg} / \mathrm{kg}$ body weight $)$ in 4 patients, and ajmaline $(0.7 \mathrm{mg} / \mathrm{kg}$ body weight $)$ in 7 patients.

\section{Electrophysiological study}

Programmed electrical stimulation was performed in all patients using up to 3 extra-stimuli at 3 different drive cycle lengths $(600,500$, and $430 \mathrm{~ms})$ delivered to the right ventricular apex. The coupling interval of the first 2 extrastimuli was not shorter than $180 \mathrm{~ms}$ and not shorter than $200 \mathrm{~ms}$ for the third extra-stimuli. In case of non-inducibility, programmed stimulation was also performed from the right ventricular outflow tract. Sustained VF was defined as VF at a cycle length $\leq 200 \mathrm{~ms}$ and lasting $\geq 30$ seconds or requiring immediate defibrillation. Sustained ventricular tachycardia (VT) was defined as VT lasting $\geq 30$ seconds or requiring termination secondary to hemodynamic instability. Non-sustained VT was defined as $>6$ consecutive ventricular complexes or VT lasting $<30$ seconds.

\section{ICD implantation}

All patients received a transvenous ICD with stored electrograms. Recorded episodes were reviewed and adjudicated as appropriate or inappropriate therapies. VF was defined at follow-up as consecutive ventricular beats recorded from the device at a cycle length of $240 \mathrm{msec}$ or 
Table I: Clinical characteristics and outcome of 18 patients with idiopathic VF

\begin{tabular}{|c|c|c|c|c|c|c|c|c|c|c|c|}
\hline \multicolumn{5}{|c|}{ Baseline Characteristics } & \multicolumn{7}{|c|}{ ICD Therapy } \\
\hline Patient & $\begin{array}{l}\text { Age } \\
\text { (years) } \\
\text { sex }\end{array}$ & CE & $\begin{array}{c}\text { Drug test to } \\
\text { exclude } \\
\text { Brugada }\end{array}$ & PES & AS & NSVT & IS & Ind S & $\begin{array}{l}\text { Time to } \\
\text { |st AS } \\
\text { (months) }\end{array}$ & $\begin{array}{l}\text { Follow- } \\
\text { up } \\
\text { (months) }\end{array}$ & $\begin{array}{c}\text { AAD } \\
\text { during } \\
\text { follow-up }\end{array}$ \\
\hline 1 & $47, M$ & Sy-VF & $\mathrm{F}$ & SMVT & 6 & 7 & - & - & 4 & 17 & sotalol \\
\hline 2 & $5 \mathrm{I}, \mathrm{F}$ & CA & $\mathrm{F}$ & $\mathrm{NI}$ & 7 & 21 & - & - & I & 19 & sotalol \\
\hline 3 & $66, M$ & CA & AJ & VF & - & - & - & - & - & 5 & - \\
\hline 4 & $70, \mathrm{~F}$ & CA & $P$ & $\mathrm{NI}$ & - & - & $6(\mathrm{af})$ & - & - & 19 & $\begin{array}{c}\text { amiodaron } \\
\mathrm{e}\end{array}$ \\
\hline 5 & $46, M$ & CA & $\mathrm{F}$ & $\mathrm{NI}$ & - & 1 & $I(a f)$ & - & - & 23 & sotalol \\
\hline 6 & $20, M$ & Sy-VF & $A$ & VF & - & - & $3(s t-l p)$ & - & - & 92 & b-blocker \\
\hline 7 & $46, \mathrm{~F}$ & $\mathrm{CA}$ & $P$ & VF & - & 120 & - & - & - & 27 & sotalol \\
\hline 8 & $40, M$ & $\mathrm{CA}$ & A & NSVT & I & - & - & - & 13 & 75 & - \\
\hline 9 & $33, M$ & Sy-VF & $\mathrm{F}$ & NSVT & - & 1 & - & - & - & 19 & - \\
\hline 10 & $26, M$ & $\mathrm{CA}$ & $\mathrm{F}$ & $\mathrm{NI}$ & I & - & $3(s t-1 p)$ & - & 7 & 9 & sotalol \\
\hline 11 & $33, \mathrm{~F}$ & $C A$ & $\mathrm{~F}$ & $\mathrm{NI}$ & - & - & - & - & - & 18 & - \\
\hline 12 & $50, \mathrm{~F}$ & $C A$ & $\mathrm{~F}$ & SMVT & 6 & - & $I(s t)$ & - & 13 & 86 & flecainide \\
\hline 13 & $25, M$ & $C A$ & $A J+P$ & NSVT & - & 1 & - & - & - & 62 & - \\
\hline 14 & $36, M$ & $\mathrm{CA}$ & AJ & $\mathrm{NI}$ & 2 & - & $2(a f)$ & 4 & 27 & 55 & sotalol \\
\hline 15 & $58, M$ & Sy-VF & AJ & $\mathrm{NI}$ & - & - & $3(\mathrm{af})$ & 2 & - & 57 & sotalol \\
\hline 16 & $44, M$ & $\mathrm{CA}$ & $F+P$ & $\mathrm{NI}$ & - & - & - & - & - & 48 & - \\
\hline 17 & $28, M$ & Sy-VF & $F$ & VF & 4 & - & $2(s t)$ & - & 18 & 55 & sotalol \\
\hline 18 & $35, M$ & Sy-VF & A & SMVT & - & - & - & - & - & 59 & - \\
\hline
\end{tabular}

CE: clinical events; CA: cardiac arrest; Sy-VF: syncope with documented self-terminating polymorphic VT or VF

PES: programmed electrical stimulation; M: male; F: female

SM: sustained monomorphic; VT: ventricular tachycardia; VF: ventricular fibrillation

NS: non sustained; NI: non inducibility; af: atrial fibrillation; st: sinus tachycardia; Ip: lead problem

AS: appropriate shock; IS: inappropriate shock; Ind S: indeterminate cause for shocks;

AAD: antiarrhythmic drug; F: flécaïnide; AJ: ajmaline; P: procainamide

less. In all patients, the ICD was programmed with only 1 ventricular fibrillation detection zone at $180 \mathrm{bpm}$ (333 msec). Only shock therapy was programmed. All patients were seen at follow-up in our centre.

\section{Statistical analysis}

All data are expressed as mean \pm standard deviation. Kaplan-Meier analysis was used to analyze time intervals until the first appropriate shock.

\section{Results}

The study cohort consisted of 13 men and 5 women (Table 1), with a mean age at the first ICD implantation of $42 \pm 14$ years (range 20 to 70 years). Baseline electrocardiograms were normal in 16 patients. One patient had a left bundle branch block and 1 had a right bundle branch block. The mean $\mathrm{QT}_{\mathrm{c}}$ for the entire population was $410 \pm 20 \mathrm{msec}$. All patients had a normal left ventricular ejection fraction $(\geq 50 \%)$ without regional wall motion abnormalities. Mean left ventricular ejection fraction (EF) was $69 \pm 8 \%$ (range 52 to $81 \%$ ). No patient had significant coronary artery disease (defined as $\geq 50 \%$ stenosis). Microscopic examination of right ventricular biopsy spec- imens in 10 patients showed no evidence of a viral, infiltrative or dysplasic process in the myocardium. Baseline electrophysiological studies were performed in all patients and showed normal sinus node, atrioventricular node and His-Purkinje function in all. Inducible sustained ventricular arrhythmias occurred in 7 patients (39\%). From these, 4 patients had sustained VF and 3 patients had sustained inducible monomorphic VT or ventricular flutter (cycle length $230 \pm 20 \mathrm{~ms}$ ). Two extrastimuli were required in 5 patients and 3 extra-stimuli in 2 patients. Non-sustained VT-VF was induced in 3 patients. No arrhythmia could be induced in 8 patients.

\section{Long-term outcome}

After a mean follow-up of $41 \pm 27$ months (ranging from 5 to 92 months), 7 of the 18 patients (39\%) with idiopathic VF had VF or sustained polymorphic VT recurrence and received appropriate shocks (Table 1). These patients experienced a total of 27 shocks; 5 of them had more than 1 episode ( 2 to 7 ). Mean time to first appropriate shock was $12 \pm 9$ months (ranging from 0.4 to 27 months). No arrhythmic storm was observed at follow-up (defined as $\geq$ 3 separate VT/VF episodes within $24 \mathrm{~h}$ ). With the use of 
stored electrograms, 6 patients had documented non-sustained polymorphic VT or VF for a total of 149 episodes (range 1 to 120). Four of them have not received any appropriate shock. These episodes were detected by the ICD but shock delivery was appropriately aborted by the non-committed function of the device. There was no relationship between the initial clinical and arrhythmic presentation and subsequent arrhythmic events recorded from the ICD at follow-up (Table 1). From the intracardiac electrograms, the arrhythmia initiation was always associated with PVCs with a mean coupling interval of $300 \pm 35 \mathrm{~ms}$. A long-short initiating sequence of VF was never observed. In 5 patients, multiple VF episodes were recorded by the ICD, all of which for a single patient were associated with the same PVC coupling interval. Since the stored electrograms in the present study were obtained from endocardial sites and were single-channel recordings, we could not assess the origin of the PVCs. The mean QTc at the time of VF recurrence was normal in all patients (mean $418 \pm 22 \mathrm{msec}$ ). The ICD effectively recognized and promptly treated all the polymorphic VT or VF recurrences and prevented the possible occurrence of sudden cardiac death. No death was reported during follow-up. Noninvasive follow-up examinations failed to detect any new structural heart disease or primary electrical disease such as long QT or Brugada syndrome.

\section{Value of electrophysiological Testing}

Programmed electrical stimulation failed to predict subsequent cardiac events. The sensitivity and specificity were 43 and 64\%, respectively. The positive and negative predictive values were also 43 and $64 \%$, respectively, that is not considered clinically useful.

\section{Causes and incidence of inappropriate shocks}

In this population, we observed a high incidence of inappropriate shocks. Eight of the 18 patients (44\%) received an inappropriate shock for a total of 21 discharges. Atrial fibrillation was responsible for $57 \%$ of them (12 episodes in 4 patients). These were older patients with a mean age of $53 \pm 14$ years. Six inappropriate shocks ( 4 patients) were triggered by sinus tachycardia. These patients were younger with a mean age of $31 \pm 13$ years. Lead malfunction caused 3 inappropriate shocks in 2 patients. Six shocks in 2 patients were classified as of unknown cause, even after careful examination of the intracardiac electrograms. These shocks were probably inappropriate since no clinical symptoms occurred during these episodes and these 2 patients already experienced inappropriate shocks for atrial fibrillation.

\section{Device follow-up}

During the follow-up period, first-time generator replacement was performed in 9 patients after a mean of $43 \pm 11$ months after initial implantation (range 21 to 58). End of life battery was the indication for replacement in 8 patients, and 1 had an ICD component failure. A second replacement was done in 2 patients. Lead replacement was also indicated in 2 patients for lead insulation fracture. Adjunctive anti-arrhythmic drugs were required in 11 patients after ICD implantation in order to control for the occurrence of appropriate or inappropriate shocks (Table 1).

\section{Discussion}

To our knowledge, this report is the first study to describe the clinical outcome of ICD patients with a diagnosis of idiopathic VF in whom the Brugada syndrome, characterized by a high recurrence rate, was systematically excluded. Since there is a wide variability in the ECG expression in individual patients with the Brugada syndrome, we performed iv administration of sodium channel blockers to unmask the ECG features of the syndrome in all our idiopathic VF patients. Two studies reported the long-term outcome of patients with idiopathic VF without the Brugada syndrome $[5,6]$. Viskin et al. [5] performed iv administration of a sodium channel blocker to unmask a concealed form of Brugada syndrome in only $15 \%$ of their idiopathic VF population, whereas Remme et al. [6] tested the effect of an iv sodium channel blocker on ECG morphology in only $30 \%$ of the patients. The unique finding of the present report is the high recurrence rate of sustained ventricular arrhythmias in this well-characterized idiopathic VF group, even after a careful systematic evaluation to exclude the presence of Brugada syndrome. After a mean follow-up of $41 \pm 27$ months, $39 \%$ of the patients received an appropriate ICD discharge for VF or polymorphic VT. In accordance with references [8,9], ICD therapy offered good protection against fatal outcome due to recurrent ventricular arrhythmias, with no mortality during the follow-up. The high recurrence rate is consistent with the relatively high recurrence rate of arrhythmic events or sudden death from the UCARE registry [10], as well as the high frequency of electrical discharges from ICDs reported in a large series of patients with idiopathic VF $[6,8,9]$. In comparison, appropriate ICD discharges have been reported in $48 \%$ of arrhythmogenic right ventricular dysplasia population [11], 40 to $56 \%$ of inducible population receiving an ICD $[12,13]$ and 23\% (7\%/year) in the hypertrophic cardiomyopathy population [14]. However, some authors have reported a more benign clinical course of their idiopathic VF population with a lower recurrence rate [15-17]. Our high ICD discharge rate might also be explained by the rapid ICD intervention for fast ventricular arrhythmia that could have been self-terminated. The definition of idiopathic VF remains problematic, since numerous studies including idiopathic VF population are heterogeneous. Indeed, 61\% (11/18 patients) of our cohort remained free of sustained arrhythmia recurrence during follow-up despite the same 
initial VF or syncopal presentation. Of note, idiopathic VF is always a diagnosis of exclusion. The patients in our cohort, as well as in other reported series, had normal hearts, as defined by clinical, non-invasive and invasive testing. Even after a careful investigation, a transient phenomenon such as a reversible localized myocardial disease, silent myocardial ischemia due to coronary artery spasm or sudden manifestation of an unknown primary electrophysiological disease could easily be missed by the clinician, and be responsible for the VF episode. Idiopathic VF is in fact an amalgam of different diseases in which the first clinical manifestation is VF. The ultimate answer probably lies in a more comprehensive approach and a more precise understanding of the molecular genetics and associated electrophysiological abnormalities in finding a specific treatment avoiding ICD implantation with its associated complications.

Anti-arrhythmic drugs have also been used in this clinical condition. Belhassen et al. [18] reported excellent results in their idiopathic VF population with EP-guided therapy using Class 1A drugs, primarily quinidine; no death occurred during a mean follow-up of $>9$ years. Recent evidence also suggests that frequent premature ventricular contractions arising from the Purkinje system are responsible for initiation of ventricular fibrillation, and can be mapped and ablated in selected patients [19]. Until another proven therapy has been assessed prospectively, the possibility of VF recurrence mandates ICD implantation as currently proposed in ICD guidelines [20].

\section{Electrophysiological findings}

The inducibility rate $(39 \%)$ of sustained VT/VF observed in our study is also in accordance with the results of the UCARE registry [10]. Of importance, programmed electrical stimulation was of limited value with a poor sensitivity and specificity. Moreover, inducibility failed to predict subsequent arrhythmic events. Our findings differ from the observed $79 \%$ average inducibility rate reported by Belhassen et al. $[17,18]$. This may be due to different patient characteristics and stimulation protocol. Our study excluded patients with Brugada syndrome known to have a high inducibility rate [21]. Compared to our stimulation protocol, Belhassen et al. [22] used up to 3 extrastimuli, 2 basic cycle lengths, 2 RV sites (first RVA, then RVOT), and repetition of extra-stimulation ( $n=10$ for double, and $n=5$ for triple) at the shortest coupling intervals that resulted in ventricular capture. Three patients had a fast monomorphic VT inducible even if the clinical presentation was aborted sudden cardiac death. This may suggest an underlying structural heart disease undetected by the current investigation. Although general cardiac function can be normal, patients might have discrete abnormalities, which are currently unidentifiable. Better risk stratification is required to identify patients who will experience recurrent VF over time. Therefore, defibrillator implantation could be seen as the primary therapy in patients with idiopathic VF, since no stratification has yet identified patients at risk of arrhythmia recurrence.

\section{ICD limitations}

The impact of multiple battery replacements over time in this young population with idiopathic VF needs to be emphasized. It is noteworthy that half the population $(9 /$ 18 patients) had an ICD replacement and 2 other patients had their transvenous lead replaced during follow-up. We also observed a high incidence of inappropriate shocks (44\%), mainly caused by atrial fibrillation and sinus tachycardia. This is well in accordance with other studies where up to $40 \%$ inappropriate discharge rates were observed even with fourth generation ICDs [23]. The addition of an anti-arrhythmic drug to decrease the incidence of inappropriate shocks was required in this group. Four patients experienced atrial fibrillation, which might indicate the presence of an associated primary electrophysiological disease also affecting the atrium. In patients with idiopathic $\mathrm{VF}$, the characteristics, is the recurrence of VF and not monomorphic VT compared to other ventricular arrhythmias. To decrease the number of inappropriate shocks, one should program a higher VF zone around 200-220 bpm and a lower monitoring zone to detect atrial arrhythmia. Third-generation ICD still have limitations and complications over time, with a significant proportion of patients having hardware-related complications or inappropriate shocks [24]. Future developments in ICD technology is needed, which will hopefully address these issues.

\section{Conclusions}

Idiopathic VF patients have a high recurrence rate of ventricular arrhythmias, even after the systematic exclusion of patients with the Brugada syndrome or other known electrical diseases from the analysis. ICD prevents sudden cardiac death in this population, but treat only the final manifestation of an unknown disease. Inappropriate shocks remain a major concern in this young population.

\section{Competing interests}

The author(s) declare that they have no competing interests.

\section{Author's contributions}

JC participated in the hypothesis generation and drafted the manuscript. PG and PB were project managers. FP participated in the final writing of the manuscript. All authors have read and approved the final version of the manuscript.

\section{Acknowledgements}

Dr Champagne was supported by a grant from the Quebec Heart Institute, Quebec City, Canada. 


\section{References}

I. Consensus statement of the joint steering committees of the unexplained cardiac arrest registry of Europe and of the idiopathic ventricular fibrillation registry of the United States: Survivors of out-ofhospital cardiac arrest with apparently normal heart. Need for definition and standardized clinical evaluation. Circulation 1997, 95:265-72.

2. Brugada P, Brugada J: Right bundle branch block, persistent ST segment elevation and sudden cardiac death: a distinct clinical and electrocardiographic syndrome. A multicenter report. J Am Coll Cardiol 1992, 20: |391-6.

3. Brugada R, Brugada J, Antzelevitch C, Kirsch GE, Potenza D, Towbin JA, Brugada P: Sodium channel blockers identify risk for sudden death in patients with ST-segment elevation and right bundle branch block but structurally normal hearts. Circulation 2000, I 01:5 I0-5.

4. Wilde AA, Antzelevitch C, Borggrefe M, Brugada J, Brugada R, Brugada P, Corrado D, Hauer RN, Kass RS, Nademanee K, Priori SG, Towbin J: Proposed diagnostic criteria for the Brugada syndrome: Consensus report. Circulation 2002, I06:25I4-9.

5. Viskin S, Fish R, Eldar M, Zeltser D, Lesh MD, Glick A, Belhassen B: Prevalence of the Brugada sign in idiopathic ventricular fibrillation and healthy controls. Heart 2000, 84:3I-6.

6. Remme CA, Wever EF, Wilde AA, Derksen R, Hauer RN: Diagnosis and long-term follow-up of the Brugada syndrome in patients with idiopathic ventricular fibrillation. Eur Heart J 2000, 22:400-9.

7. Chen Q, Kirsch GE, Zhang D, Brugada R, Brugada J, Brugada P, Potenza D, Moya A, Borggrefe M, Breithardt G, Ortiz-Lopez R, Wang Z, Antzelevitch C, O'Brien RE, Schulze-Bahr E, Keating MT, Towbin JA, Wang Q: Genetic basis and molecular mechanism for idiopathic ventricular fibrillation. Nature 1998, 392:293-6.

8. Meissner MD, Lehmann MH, Steinman RT, Mosteller RD, Akhtar M, Calkins H, Cannom DS, Epstein AE, Fogoros RN, Liem LB, Marchlinski FE: Ventricular fibrillation in patients without significant structural heart disease: a multicenter experience with implantable cardioverter-defibrillator therapy. J Am Coll Cardiol 1993, 2 1: 1406-12.

9. Fan W, Peter CT: Survival and incidence of appropriate shocks in implantable cardioverter defibrillator recipients who have no detectable structural heart disease. CEDARS investigators. Am J Cardiol 1994, 74:687-90.

10. Priori SG, Paganini V, Boccalatte L, Schwartz PJ: Idiopathic ventricular fibrillation: From a collection of clinical cases to a prospective evaluation. The U-CARE steering committee. Unexplained Cardiac Arrest Registry of Europe. G Ital Cardiol 1995, 25:149-58.

II. Corrado D, Leoni L, Link MS, Della Bella P, Gaita F, Curnis A, Salerno JU, Igidbashian D, Raviele A, Disertori M, Zanotto G, Verlato R, Vergara G, Delise $P$, Turrini $P$, Basso $C$, Naccarella F, Maddalena F, Estes NA, Buja G, Thiene G: Implantable cardioverter-defibrillator therapy for prevention of sudden death in patients with arrhythmogenic right ventricular cardiomyopathy/dysplasia. Circulation 2003, 108:3084-91.

12. Costeas XF, Link MS, Foote CB, Homoud MK, Wang PJ, Estes NA: Predictors of ventricular tachycardia recurrence in 100 patients receiving tiered therapy defibrillators. Clin Cardiol 2000, 23:852-6.

13. Menon V, Steinberg JS, Akiyama T, Beckman K, Carillo L, Kutalek S: Implantable cardioverter defibrillator discharge rates in patients with unexplained syncope, structural heart disease, and inducible ventricular tachycardia at electrophysiologic Study. Clin Cardiol 2000, 23:195-200.

14. Maron BJ, Shen WK, Link MS, Epstein AE, Almquist AK, Daubert JP, Bardy GH, Favale S, Rea RF, Boriani G, Estes NA, Spirito P: Efficacy of implantable cardioverter-defibrillators for the prevention of sudden death in patients with hypertrophic cardiomyopathy. N Engl J Med 2000, 342:365-73.

15. Crijns HJ, Wiesfeld AC, Posma JL, Lie KI: Favourable outcome in idiopathic ventricular fibrillation with treatment aimed at prevention of high sympathetic tone and suppression of inducible arrhythmias. Br Heart J 1995, 74:408- 12.

16. Mewis C, Kuhlkamp V, Spyridopoulos I, Bosch RF, Seipel L: Late outcome of survivors of idiopathic ventricular fibrillation. $\mathrm{Am} J$ Cardiol 1998, 81:999-1003.
17. Belhassen B, Shapira I, Shoshani D, Paredes A, Miller H, Laniado S: Idiopathic ventricular fibrillation: Inducibility and beneficial effects of class I antiarrhythmic agents. Circulation I987, 75:809-16.

18. Belhassen B, Viskin S, Fish R, Glick A, Setbon I, Eldar M: Effects of electrophysiologic-guided therapy with Class IA antiarrhythmic drugs on the long-term outcome of patients with idiopathic ventricular fibrillation with or without the Brugada syndrome. J Cardiovasc Electrophysiol 1999, I 0: | 30 |- | 3 | 2.

19. Haissaguerre M, Shoda M, Jais P, Nogami A, Shah DC, Kautzner J Arentz T, Kalushe D, Lamaison D, Griffith M, Cruz F, de Paola A, Gaita F, Hocini M, Garrigue S, Macle L, Weerasooriya R, Clementy J: Mapping and ablation of idiopathic ventricular fibrillation. Circulation 2002, 106:962-967.

20. Priori SG, Aliot E, Blomstrom-Lundqvist C, Bossaert L, Breithardt G Brugada P, Camm AJ, Cappato R, Cobbe SM, Di Mario C, Maron BJ, McKenna WJ, Pedersen AK, Ravens U, Schwartz PJ, Trusz-Gluza M, Vardas P, Wellens HJ, Zipes DP: Task Force on Sudden Cardiac Death of the European Society of Cardiology. Eur Heart J 200 I, 22:1374-|450.

21. Brugada J, Brugada R, Brugada P: Right bundle-branch block and ST-segment elevation in leads VI through V3: a marker for sudden death in patients without demonstrable structural heart disease. Circulation 1998, 97:457-60

22. Belhassen B, Shapira I, Sheps D, Laniado S: Programmed ventricular stimulation using up to two extrastimuli and repetition of double extrastimulation for induction of ventricular tachycardia: A new highly sensitive and specific protocol. $\mathrm{Am} J$ Cardiol 1990, 65:615-622

23. Washizuka T, Chinushi M, Tagawa M, Kasai H, Watanabe H, Hosaka Y, Yamashita F, Furushima H, Abe A, Watanabe H, Hayashi J, Aizawa $Y$ : Inappropriate discharges by fourth generation implantable cardioverter defibrillators in patients with ventricular arrhythmias. Jpn Circ J 2001, 65:927-30.

24. Nunain SO, Roelke M, Trouton T, Osswald S, Kim YH, Sosa-Suarez G, Brooks DR, McGovern B, Guy M, Torchiana DF, Vlahakes G], Garan $H$, Ruskin JN: Limitations and late complications of third-generation automatic cardioverter-defibrillators. Circulation 1995, $91: 2204-13$.

\section{Pre-publication history}

The pre-publication history for this paper can be accessed here:

http://www.biomedcentral.com/1741-7015/3/1/prepub

Publish with Bio Med Central and every scientist can read your work free of charge

"BioMed Central will be the most significant development for disseminating the results of biomedical research in our lifetime. "

Sir Paul Nurse, Cancer Research UK

Your research papers will be:

- available free of charge to the entire biomedical community

- peer reviewed and published immediately upon acceptance

- cited in PubMed and archived on PubMed Central

- yours - you keep the copyright

Submit your manuscript here:

http://www.biomedcentral.com/info/publishing_adv.asp
BioMedcentral 